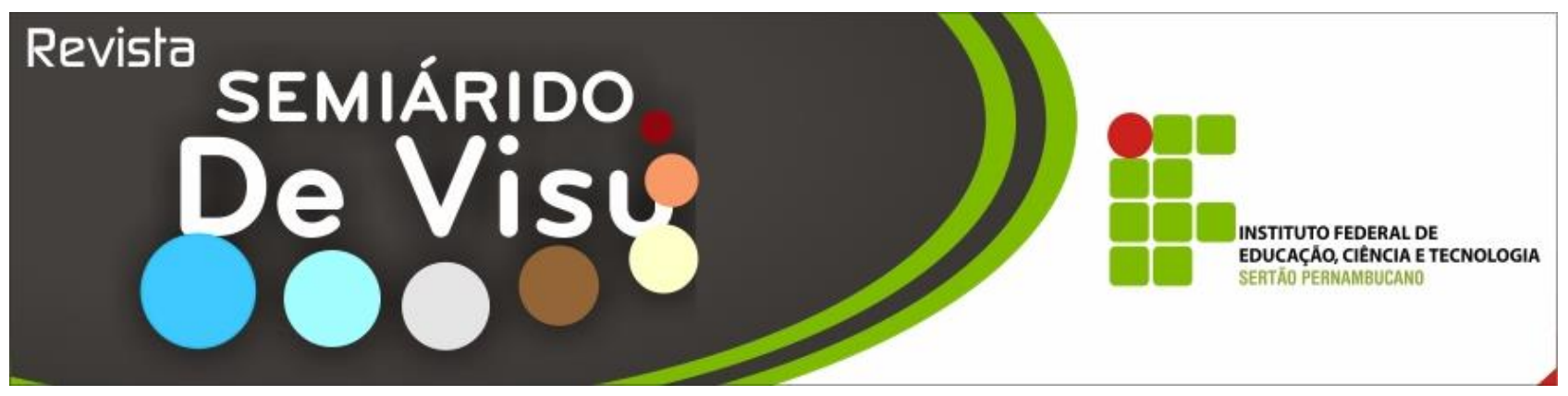

\title{
Perspectivas e desafios do arranjo produtivo local (APL) da apicultura no Município de Ouricuri, Estado Pernambuco
}

\author{
Rosangela Maria Pereira ${ }^{1}$; Társio Thiago Lopes Alves ${ }^{2}$; Janeanne Nascimento Silva ${ }^{3}$; Iris Maria \\ Silva ${ }^{4}$; Sergio Eduardo de Holanda Alencar ${ }^{5}$; Antônio Alves de Souza ${ }^{6}$; Marina Barbosa da Silva ${ }^{7}$ \\ 1, 2, 4, 5, 6, 7 Instituto Federal do Sertão Pernambucano - Campus Ouricuri. Ouricuri - Pernambuco - Brasil. \\ ${ }^{3}$ Faculdade de Juazeiro do Norte (FJN). Juazeiro do Norte - Ceará - Brasil. \\ E-mails: rosapereira069@gmail.com ${ }^{1}$; tarsiothiago@ hotmail.com ${ }^{2}$; jane_anne82@yahoo.com.br ${ }^{3}$; irismaria230789@ hotmail.com ${ }^{4}$; \\ aalvesdesouza6@gmail.com ${ }^{6}$; marina2132009@gmail.com
}

\begin{abstract}
RESUMO: A Apicultura na Região do Sertão do Araripe expandiu sua atividade a partir da ampliação do mercado do Piauí, fato justificado por essa região estar próxima aos principais arranjos produtivos de mel piauienses. Objetivouse estudar o APL da Apicultura no Município de Ouricuri- PE. Inicialmente, foi feito um estudo prévio em Associação de Apicultores, Sindicatos dos Trabalhadores Rurais, Entidades Governamentais e Não-Governamentais sobre as localidades que tinham a apicultura como atividade rural e, posteriormente, realizaram-se visitas aos apicultores identificados, aplicando questionário socioeconômico, tecnológico e associativismo, além da Metodologia para Resolução de Problemas. Observou-se que $100 \%$ dos entrevistados criam abelhas do gênero Apis, entretanto, apenas $13 \%$ têm a apicultura como atividade principal e $23 \%$ consorcia abelhas com ovinocaprinos. Em relação a perdas de colônias, 90\% tiveram perdas em 2014 e $73 \%$ também tiveram perdas em anos anteriores, de maneira que 100\% alegaram problemas com a estiagem. Portanto, o APL da Apicultura em Ouricuri encontra-se em dificuldades devido a perdas de colônias, promovendo um desestímulo dos apicultores, além de baixa produtividade.
\end{abstract}

Palavras-chave: Abelha; Atividade Apícola; Mel

\section{Perspectives and challenges of local productive arrangement (APL) of apiculture in Ouricuri, Pernambuco}

\begin{abstract}
The Apiculture in the region of Sertão do Araripe expanded its activities from the enlargement of the market of Piauí, this fact is justified by the proximity of this region to the main productive arrangements of honey in Piauí. It was aimed at studying the APL of the Apiculture in Ouricuri, Pernambuco. Initially, a previous study was carried out with a beekeepers' association, rural workers unions, governmental and non-governmental organizations about the places which had the apicultural activity as a rural activity and after that, visits to the identified beekeepers applying the socio-economic, technologic questionnaire, besides the solving problems methodology. It was observed that $100 \%$ of the interviewed ones raise bees of the Apis gender, however, only $13 \%$ have the apiculture as a main activity and 23\% associates bees with ovines and caprines . In relation to the losing of colonies, $90 \%$ had losses in 2014 and $73 \%$ also had losses in previous years, however, $100 \%$ claimed problems with drought. Therefore, the APL of apiculture in Ouricuri is in problem due to the losing of colonies, promoting a disincentive of the beekeepers, besides the low productivity.
\end{abstract}

Keywords: Bee, Apicultural activity and Honey 
Introdução

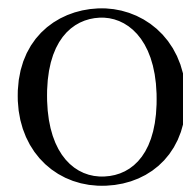

$\mathrm{s}$ Arranjos Produtivos Locais (APLs) ou Sistemas Locais de Produção (SLPs) são termos genéricos utilizados para representar um conjunto de casos particulares, tais como: aglomerações de empresas, parques tecnológicos, millieux inovadores, sistema nacional de inovação, clusters, distritos industriais, redes empresariais, entre outros. Estes são alguns dos termos encontrados amplamente na literatura para designar um agrupamento de empresas. Essa vasta terminologia, que muitas vezes é usada para indicar o mesmo fenômeno, vem sendo utilizada nos últimos anos em vários ambientes (acadêmicos, empresariais, políticos), em que esteja presente a preocupação com temas como competitividade empresarial, desenvolvimento regional e políticas industriais. A importância desses agrupamentos de produtores cresceu de modo significativo no período recente, especialmente depois de meados da década de 70 quando ressurgiram no cenário internacional os chamados distritos industriais italianos. Nesses casos, a aglomeração de empresas de pequeno e médio portes proporcionou aos produtores ganhos expressivos de competitividade que se traduziram numa expansão do comércio internacional (Bagnasco, 1999; Becattini, 1999; Gurisatti, 1999; Coró, 1999; Cassia, 1999).

Para compreender como a Apicultura no Araripe se configura como um APL, é preciso enxergá-la como mais do que uma atividade econômica que aglomera vários empreendimentos em um mesmo local; é necessário analisar como as "[...] forças sociais cooperam com as econômicas" (Marshall, 1996). Os arranjos produtivos agregam "[...] trajetórias históricas de construção de identidades e de formação de vínculos territoriais" (Lastres; Cassiolato, 2004) relacionadas com os atores e com as atividades sociais, culturais, políticas e econômicas comuns. Esses APL desenvolveram elementos que propiciaram sua integração e seu crescimento como interação, cooperação, capital social e confiança entre os atores.

A atividade apícola na Região do Sertão do Araripe expandiu sua atividade a partir da ampliação do mercado do Piauí, fato justificado por essa região estar próxima aos principais arranjos produtivos de mel piauienses (Araújo, 2006). Essa proximidade permitiu aos apiários piauienses migrarem para a Chapada do Araripe, principalmente para a área que fica no território pernambucano. Essa característica do manejo apícola no Nordeste é fundamental para diferenciá-la da produção no Sul e destacá-la como produto orgânico, inclusive por ser a qualidade do mel considerada excelente e por promover vínculos sociais mais solidários (Paula Neto; Almeida Neto, 2006). No Sul, a alimentação é em grande parte artificial devido aos períodos de inverno.

$\mathrm{O}$ trajeto histórico da apicultura nessa região culmina na formação do espaço geográfico, localizado no Sertão de Pernambuco, sendo constituída por dez municípios: Araripina, Bodocó, Granito, Ipubi, Moreilândia, Ouricuri, Santa Cruz, Santa Filomena e Trindade, abrangendo uma área de $11.969,5 \mathrm{Km}^{2}$, e conta com uma população de 218.538 habitantes (IBGE, 2010). Essa demarcação da escala espacial do Araripe é analisada como uma definição do Governo de que o local seria, então, uma apreensão sobre um emaranhado de conceitos e significados que algumas vezes se tornam contraditórios, mesmo remetendo à ideia de lugar ou espaço territorial limitado (Vainer, 2002). Portanto, quando observada a dinâmica histórica, esta é passível de modificação, ou mesmo de uma nova definição pelas pessoas e pela cultura, elementos importantes na identidade do lugar.

Objetivou-se, com a pesquisa, estudar o Arranjo Produtivo Local (APL) da Apicultura no Município de Ouricuri, PE.

\section{Material e Métodos}


Inicialmente, foi realizado um estudo prévio em Associação de Apicultores, Sindicatos dos Trabalhadores Rurais, Entidades Governamentais e NãoGovernamentais sobre os distritos que tinham a apicultura como atividade rural no município de Ouricuri-PE.

Posteriormente, foram realizadas visitas aos apicultores dos distritos identificados e aplicou-se um questionário socioeconômico, tecnológico (uso de tecnologias, produção, manejo, comercialização, perfil do consumidor) e Associativismo, além da Metodologia para Resolução de Problemas (MRP), cujo processo fundamenta-se em duas estruturas básicas: estrutura de comportamento e estrutura de conteúdo. A estrutura de comportamento refere-se a um modelo sistemático de se comportar em grupo. Em decorrência disso, todo o processo das duas fases de trabalho se intercala e é desenvolvido de modo a facilitar maior participação e a obtenção do sucesso do resultado final: Fase de Personalização, em que é dada ênfase ao trabalho individual de resolução de problemas; Fase de Colaboração, em que ocorre a troca de informações, atitudes e sentimentos sobre o problema e sua resolução. Nessa fase, cada participante trabalha de forma colaborativa, como membro do grupo.

A estrutura de conteúdo refere-se às etapas:

Gênese - O problema é identificado seguindo três passos. No primeiro, identificase a área geral do problema. No segundo, descreve-se a situação atual, considerando-se aspectos positivos e negativos da área problema. No terceiro passo, descreve-se a situação desejada. Por problema, entende-se a diferença entre a situação atual e a situação desejada.
Diagnóstico - O problema é compreendido em termos de forças restritivas e forças impulsoras. Compreende-se força restritiva uma condição, pessoa ou diretriz, que restringe a mudança da situação atual para a situação desejada. É uma força que precisa ser diminuída ou removida para que o problema seja resolvido. Força impulsora é uma condição, pessoa ou diretriz que, se fortalecida, possibilitará a solução do problema.

Análise - Nessa etapa, listam-se as linhas de ação a serem adotadas, as responsabilidades pela sua realização e agendamento.

Entende-se, portanto, por problema a diferença entre a situação "atual e situação desejada". A descrição dos dados foi realizada por meio de estatística descritiva, além da construção de gráficos, cálculos de médias e porcentagens dentro de cada item avaliado.

\section{Resultados e Discussão}

Diante dos resultados, observou-se que 100\% dos entrevistados criam abelhas do gênero Apis, entretanto, apenas $13 \%$ têm a apicultura como atividade principal na propriedade e $23 \%$ consorcia abelhas com ovinocaprinos (Figura 01). A maioria (73\%) tem pequena propriedade rural, com até 20 hectares, tendo, ao redor do apiário, vizinhos que exercem a atividade de agricultura (77\%) e $18 \%$ residências. Esses resultados vão de encontro a trabalhos realizados no Nordeste Brasileiro, os quais mostram que a maioria dos produtores tem baixa escolaridade e a apicultura é uma atividade complementar na propriedade (Arruda et al., 2011; Leão et al., 2012; Ribeiro et al., 2012). 
Figura 01. Detalhe das atividades desenvolvidas nas propriedades rurais. IFSertão-PE. Ouricuri, 2015 .

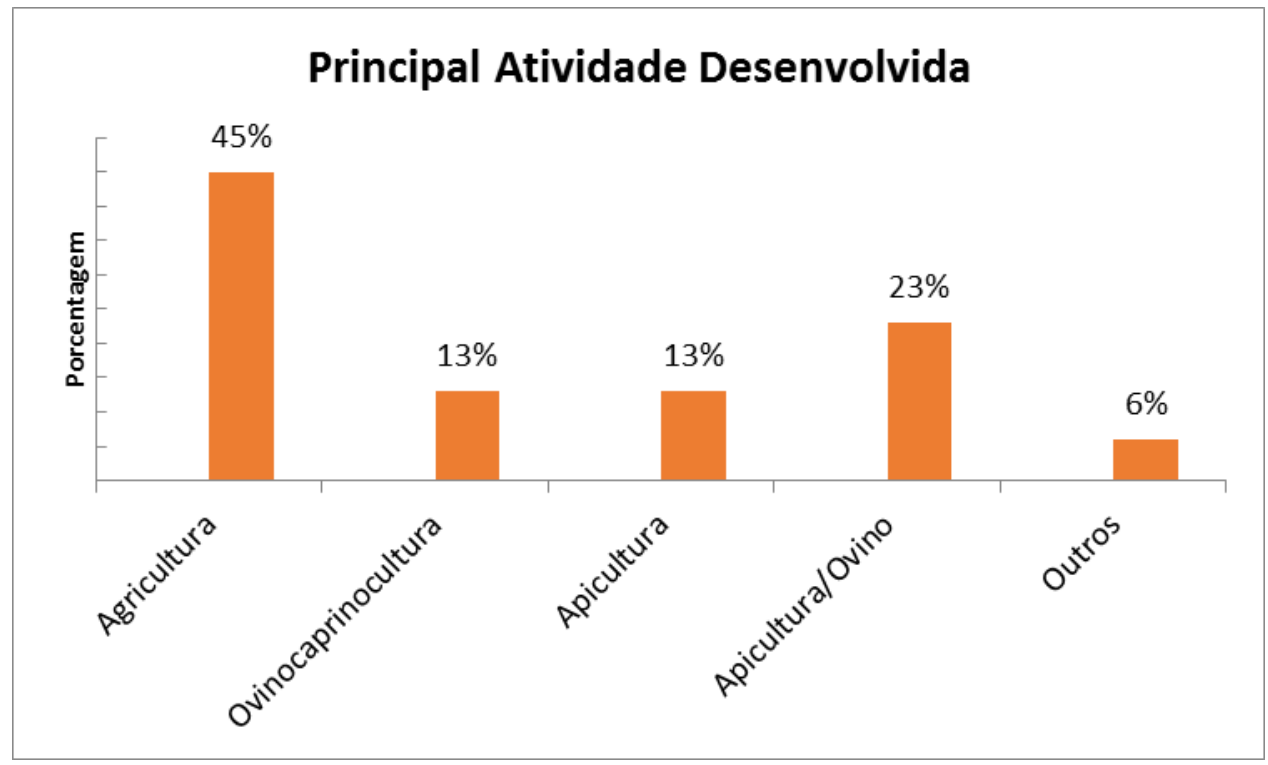

Fonte: Pesquisa direta.

Todos os entrevistados têm até 50 colônias povoadas, especificamente entre $20 \mathrm{e}$ 30 colônias, com uma produtividade média de $15 \mathrm{Kg}$ de mel/colônia/ano (41\%) e $36 \%$ com produtividade entre 16 e $20 \mathrm{Kg}$ de mel/colônia/ano (Figura 2). Em relação a perdas de colônias, $90 \%$ tiveram perdas em
2014 (Figura 03) e 73\% também tiveram perdas em anos anteriores, mais precisamente entre 2010 e 2013, chegando até $50 \%$ de perdas em relação às colônias povoadas, de maneira que $100 \%$ alegaram problemas com a estiagem.

Figura 02. Produtividade média de mel no APL da Apicultura em Ouricuri. IF Sertão-PE. Ouricuri, 2015.

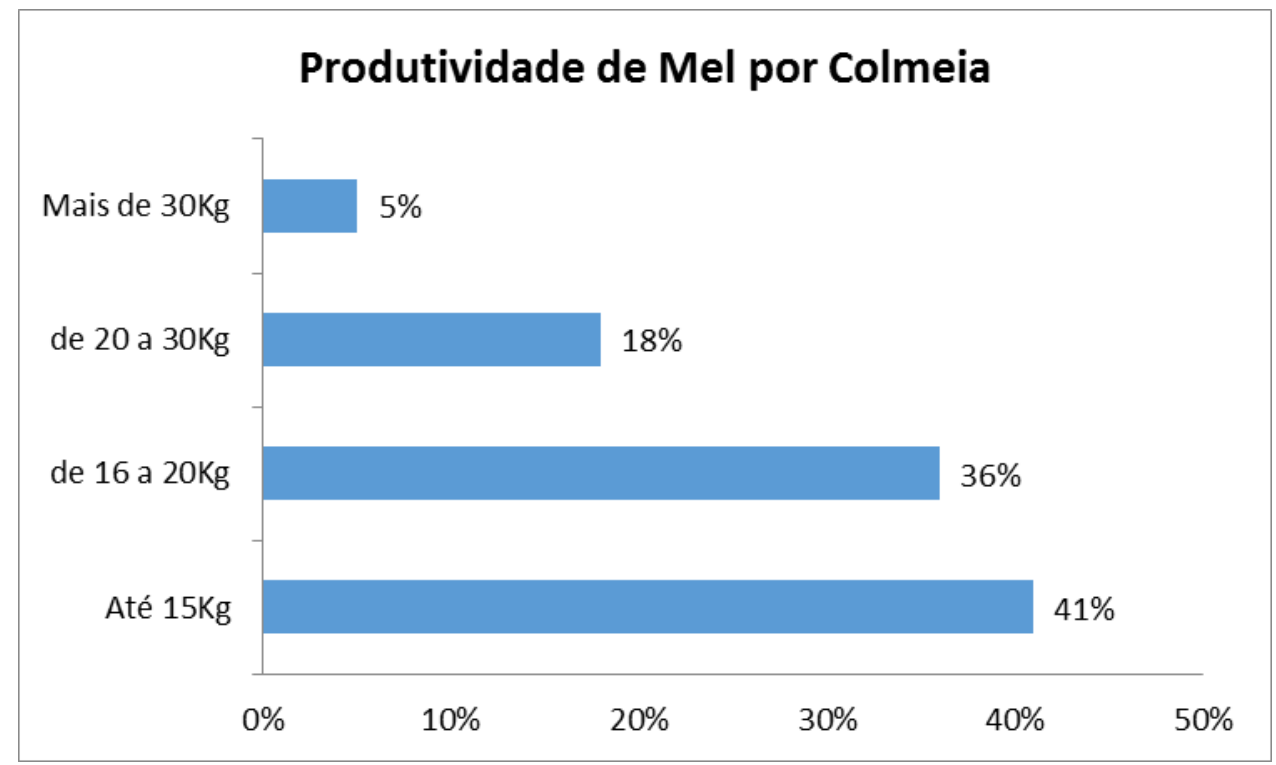

Fonte: Pesquisa direta. 
Figura 03. Perdas de colônias em 2014 no APL da Apicultura em Ouricuri. IF Sertão-PE. Ouricuri, 2015.

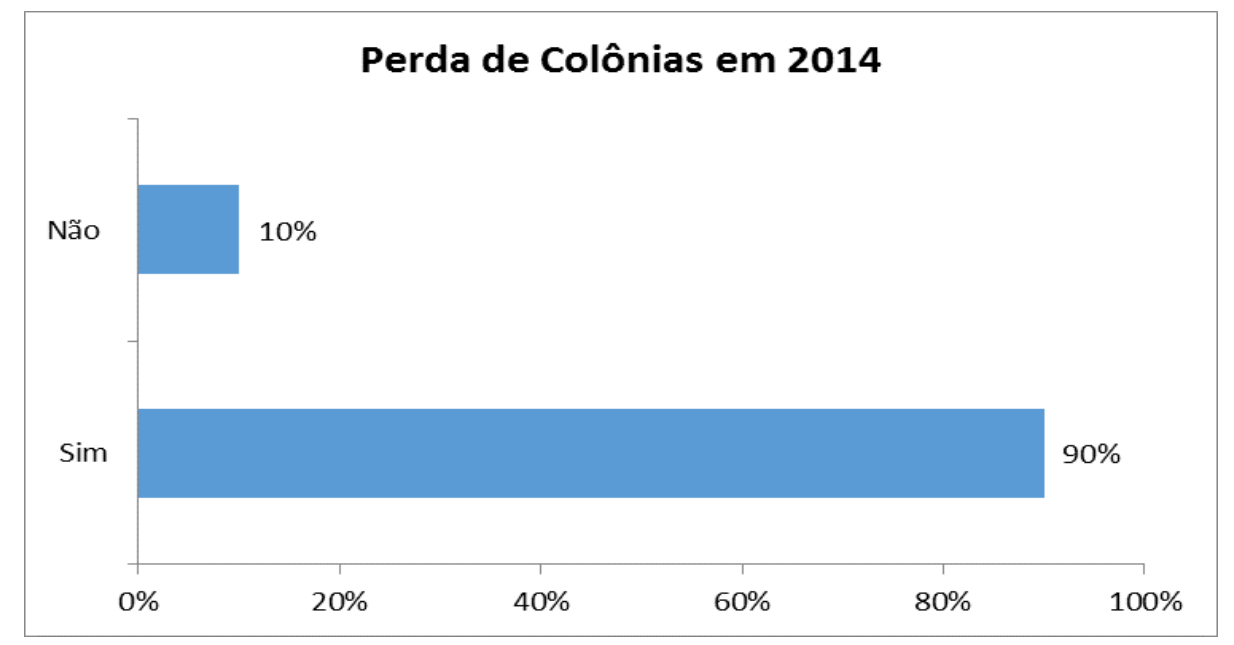

Fonte: Pesquisa direta.

Em relação ao consumo de mel, $32 \%$ consomem mel 1x por mês e de 2 a $3 x$ por semana (Figura 04) e 23\% apenas 1x no ano, todavia $73 \%$ deste consumo são feitos na forma de mel in natura (Figura 05), mostrando o baixo consumo de mel por parte dos entrevistados. Martins et al., (2010), estudando o perfil do consumidor no Estado do Ceará encontrou que $56,1 \%$ consomem eventualmente o alimento, $16,3 \%$ mensalmente, $12,9 \%$ semanalmente e $14,8 \%$ apresentam um consumo diário do mel de abelhas. Os mesmos autores identificaram que a forma de consumo mais usual pelos entrevistados é como remédio 52\%, 23,1\% como alimento e $24,9 \%$ usam o mel como remédio e alimento. Resultados semelhantes foram encontrados por Cheung \& Gerber (2009), analisando os comportamentos de comensais do Estado de Santa Catarina e Costa Júnior et al., (2006), estudando o comportamento do consumidor de mel de abelha nas cidades cearenses de Crato e $\begin{array}{lll}\text { Juazeiro do Norte. } & \text { do }\end{array}$

Figura 04. Frequência de Consumo de Mel no APL da Apicultura em Ouricuri. IF Sertão-PE. Ouricuri, 2015.

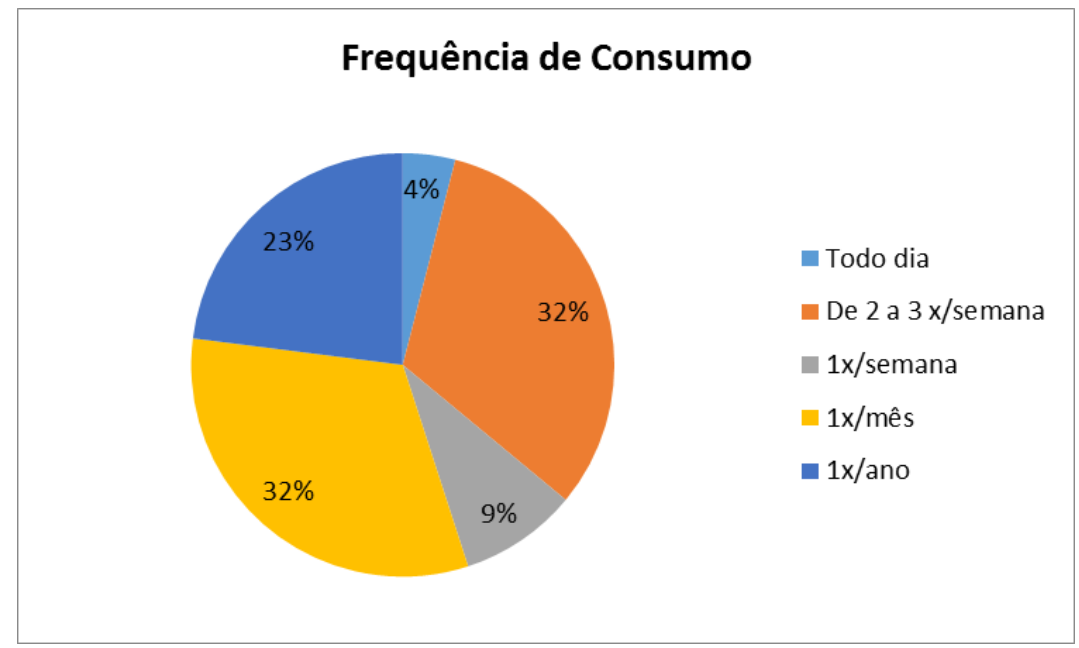

Fonte: Pesquisa direta. 
Figura 05. Forma de Consumo de Mel no APL da Apicultura em Ouricuri. IF Sertão-PE. Ouricuri, 2015.

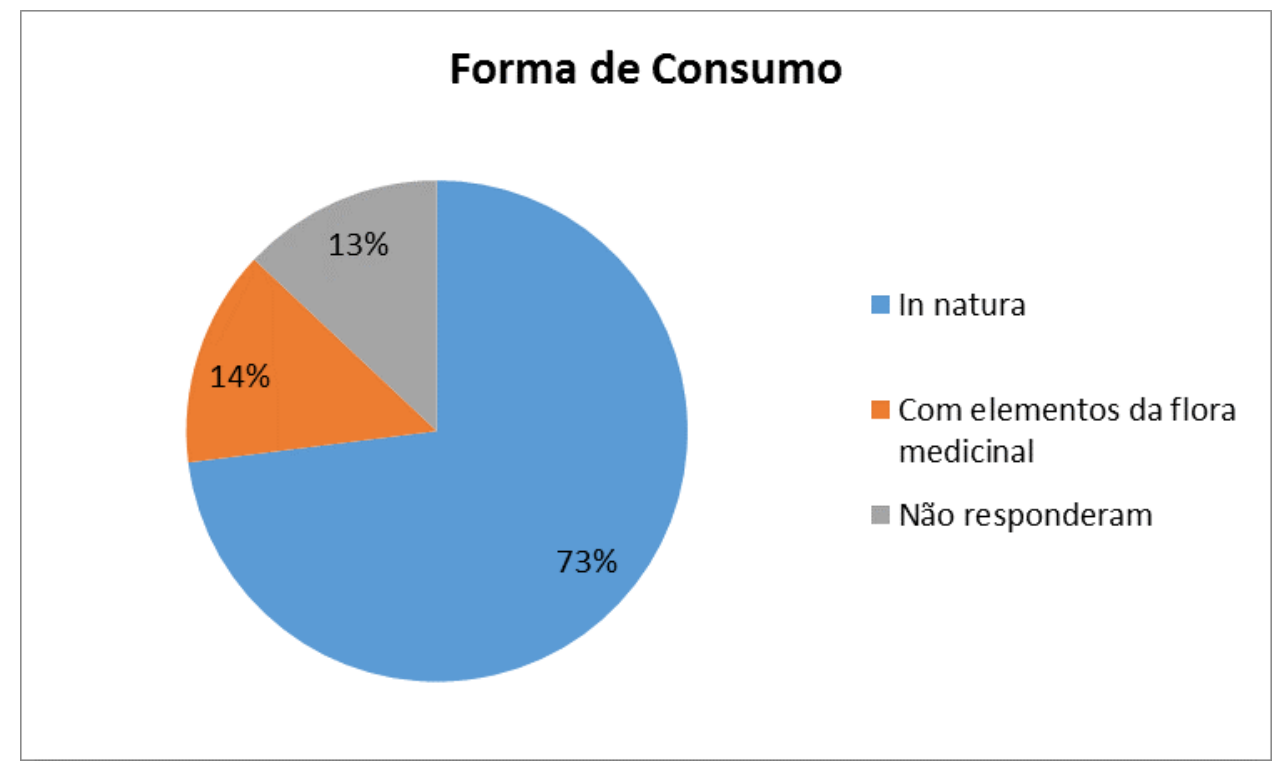

Fonte: Pesquisa direta.

\section{Conclusões}

Conclui-se que a o Arranjo Produtivo Local (APL) da Apicultura em Ouricuri-PE encontra-se em dificuldades devido a perdas de colônias, promovendo um desestímulo dos apicultores, além de baixa produtividade.

O consumo de mel por parte dos apicultores é pouco, dificultando a comercialização no mercado local.

\section{Referências Bibliográficas}

ARAÚJO, Tarcisio Patricio (Org.). Demanda por microcrédito em três arranjos produtivos de Pernambuco: Apicultura, bacia leiteira e caprinocultura. Recife: Fundação Joaquim Nabuco: Massangana, 2006.

ARRUDA, J. B. F. ; BOTELHO, B. D. ; CARVALHO, T. C. Diagnóstico da cadeia produtiva da apicultura: um estudo de caso.

In: ENCONTRO NACIONAL DE ENGENHARIA DE PRODUÇÃO. 31.,
2011, Belo Horizonte. Anais... Belo Horizonte: ABEPRO, 2011.

BAGNASCO, Arnaldo. "Desenvolvimento regional, sociedade local e economia difusa". In: COCCO, Giuseppe; URANI, André; GALVÃO, Alexandre Patez (orgs). Empresários e Empregos nos Novos Territórios Produtivos: o caso da terceira Itália. Rio de Janeiro: DP\&A, 1999.

BARRETO, L. M. R. C.; FUNARI, S. R. C.; ORSI, R. O. Pólen apícola: perfil da produção no Brasil. In: CONGRESSO DE APICULTURA DEL MERCOSUR, 1. 2005, Punta Del Este. Anais... Punta Del Este: 2005. 20p.

BEGATINI, Giacomo. "Os distritos industriais na Itália". In: COCCO, Giuseppe; URANI, André; GALVÃO, Alexandre Patez (org's). Empresários e Empregos nos Novos Territórios Produtivos: o caso da terceira Itália. Rio de Janeiro: DP\&A, 1999.

BRASIL. Cadeias Produtivas de Flores e Mel. Ministério da Agricultura, Pecuária e Abastecimento - MAPA/Secretaria de 
Política Agrícola. Cord. BUAINAIN, A. M. e BATALHA, M. O. v. 9. 140p. 2007.

CASSIA, Giuseppe. "Modelos empresariais e figuras do trabalho no nordeste da Itália". In: COCCO, Giuseppe; URANI, André; GALVÃO, Alexandre Patez (org's). Empresários e Empregos nos Novos Territórios Produtivos: o caso da terceira Itália. Rio de Janeiro: DP\&A,1999.

CHEUNG, T. L; GERBER, R. M; Consumo de mel de abelhas: análise dos comportamentos de comensais do estado de Santa Catarina. Informações Econômicas, SP, v.39, n.10, out. 2009.

CORÓ, Giancarlo. "Distritos e sistemas de pequena empresa na transição". In: COCCO, Giuseppe; URANI, André; GALVÃO, Alexandre Patez (org's). Empresários e

Empregos nos Novos Territórios Produtivos: o caso da terceira Itália. Rio de Janeiro: DP\&A, 1999.

COSTA JÚNIOR, M. P.; SILVA, L. P.; SOUSA, E. P. Comportamento do consumidor de mel de abelha nas cidades cearenses de Crato e Juazeiro do Norte. In: CONGRESSO DA SOCIEDADE BRASILEIRA DE ECONOMIA E SOCIOLOGIA RURAL, 44., 2006, Fortaleza - CE. Anais... Fortaleza - CE: SOBER, 2006. CD-ROM.

DA SILVA, N. R. Aspectos do Perfil e do Conhecimento de Apicultores Sobre Manejo e Sanidade da Abelha Africanizada em Regiões de Apicultura de Santa Catarina. 2004. 117 f. Dissertação (Mestrado em Agroecossistemas) - Universidade Federal de Santa Catarina, Florianópolis, 2004.

IBGE - Instituto Brasileiro de Geografia e Estatística. Produção da pecuária municipal 2009. 2010.

JÚNIOR, M.; SILVA, L. \& SOUSA, E. (2006). Comportamento do consumidor de mel de abelha nas cidades Cearenses de Crato e Juazeiro do Norte. Anais do XILV Congresso da Sociedade de Economia e Sociologia Rural, Fortaleza.

LASTRES, Helena M. M.; CASSIOLATO, José E. (Coord.). Glossário de Arranjos e Sistemas Produtivos e Inovativos. RedeSist, 2004. Disponível em: <http://www.ie.ufrj.br/redesist/P4/Ampli/Glos s\%E1rio\%20RedeSist. pdf $>$. Acesso em: 12 março 2009.

LEÃO, E. L. S.; MOUTINHO, L. M. G.; XAVIER, M. G. P. Condicionantes de crescimento arranjo produtivo local de apicultura na Região do Araripe, Pernambuco. RACE, Unoesc,v. 11, n. 1 Edição Especial Agronegócios, p. 75-102, jan./jun. 2012.

MARCHINI, L.C., G.S. SODRÉ e A.C.C.C. MORETI. Mel brasileiro: Composição e normas. Ribeirão Preto, A.S.P., 131p. 2004.

MARSHALL, Albert. Princípios de Economia. São Paulo: Nova Cultural, 1996.

MARTINS, $\quad$ F.F.F.; $\quad$ MARTINS, F.F.; PEREIRA, J. O. P.; ALENCAR, T. C. S. B. D.; CARVALHO, L.S. ; MACEDO, S.F.L. ; FARIAS, K. C. ; PAULA, C.M. Perfil do consumo de mel de abelhas africanizadas em cidades do interior do estado do Ceará. In: V CONNEPI - Congresso de Pesquisa e Inovação da Rede Norte e Nordeste de Educação Tecnológica, 2010, Maceió. Anais do V CONNEPI - Congresso de Pesquisa e Inovação da Rede Norte e Nordeste de Educação Tecnológica, 2010.

MINISTERIO DE MINAS E ENERGIA. Projeto cadastro de fontes e abastecimento por água subterrânea no estado de Pernambuco: Diagnóstico do Município de Ouricuri. Recife. 32p. 2005.

OURICURI. Disponível em:< http://pt.wikipedia.org/wiki/Ouricuri>. Acesso em: 07 março 2013. 
PASIN, V. L. E.; TERESO, M. J. A.; BARRETO, L. M. R. C. Análise da produção e comercialização de mel natural no Brasil no período de 1999 a 2010. Revista Agroalimentaria. v. 18, n.34, p.29-42. 2012.

PAULA NETO, Francisco Leandro de.; ALMEIDA NETO, Raimundo Moreira. Apicultura nordestina: principais mercados, riscos e oportunidades. Fortaleza: Banco do Nordeste do Brasil, 2006. 78 p. (Série Documentos do ETENE, n. 12). Disponível em:

<http://www.bnb.gov.br/projwebren/exec/livr oPDF.aspx?cd_livro=5>. Acesso em: 22 março 2010.

RIBEIRO, K. A.; NASCIMENTO, D. C.; CASSUNDÉ JUNIOR, N. F.; MORATO, J. A. Q.; Arranjo produtivo local (APL) como estratégia de potencializar as fronteiras mercadológicas do apicultor no perímetro de irrigação Senador Nilo Coelho em PetrolinaPE. Revista de Gestão, Finanças e Contabilidade, UNEB, Salvador, v. 3, n. 2, p. 99-120, maio/ago., 2013.

SEBRAE. Serviço Brasileiro de Apoio as Micro e Pequenas Empresas. Boletim Setorial do Agronegócio: Apicultura. Recife.15p. 2011.

SOUZA, D. C. Apicultura Orgânica: Alternativa para Exploração da Região do Semi-Árido Nordestino. In: CONGRESSO BRASILEIRO DE APICULTURA, 14., 2002, Campo Grande. Anais... Campo Grande: Confederação Brasileira de Apicultura, 2002. p.133-135.

VAINER, Carlos Bernardo. As escalas do poder e o poder das escalas: $\mathrm{O}$ que o pode $\mathrm{O}$ poder local? In: Planejamento e Território: ensaios sobre a desigualdade. Cadernos do IPPUR/UFRJ, Rio de Janeiro: UFRJ/IPPUR, ano 15, n. 2, ago./dez. 2001; ano 16, n. 1 jan./jul. 2002. 